\title{
Molecular machines governing exocytosis of synaptic vesicles
}

Reinhard Jahn ${ }^{1} \&$ Dirk Fasshauer ${ }^{2}$

Calcium-dependent exocytosis of synaptic vesicles mediates the release of neurotransmitters. Important proteins in this process have been identified such as the SNAREs, synaptotagmins, complexins, Munc18 and Munc13. Structural and functional studies have yielded a wealth of information about the physiological role of these proteins. However, it has been surprisingly difficult to arrive at a unified picture of the molecular sequence of events from vesicle docking to calcium-triggered membrane fusion. Using mainly a biochemical and biophysical perspective, we briefly survey the molecular mechanisms in an attempt to functionally integrate the key proteins into the emerging picture of the neuronal fusion machine.

$\mathrm{E}$ xocytosis and recycling of synaptic vesicles define how much transmitter is released from nerve terminals during incoming action potentials (Fig. 1). Under resting conditions, synaptic vesicles are stored in the cytoplasm of the nerve terminal, with some of them attached to specialized sites at the presynaptic plasma membrane termed active zones. Active zones are composed of unique multidomain proteins that provide a scaffold for vesicle docking and participate in activating the release apparatus, referred to as priming. Priming probably involves several reactions, including some requiring metabolic energy. Docked and primed vesicles (termed readily releasable pool) are ready to go, and some do so spontaneously, with the transmitter released by a single vesicle giving rise to a miniature postsynaptic potential. When an action potential arrives, voltage-gated calcium channels open, with the resulting calcium influx stimulating the rate of exocytosis more than 100,000 fold in a highly cooperative manner (for review see ref. 1).

During the past two decades, the key proteins mediating neuronal exocytosis have been identified. Many of them belong to structurally conserved protein families including the SNAREs, Rab proteins, Sec1/ Munc18-like (SM) proteins, and a group of tethering proteins termed CATCHR (complex associated with tethering containing helical rods) proteins. Apparently, they form the core of an ancient intracellular fusion machine that diversified during evolution to adapt to the needs of specialized compartments. Neuronal exocytosis constitutes one of such adaptations, and specific regulatory proteins such as synaptotagmins and complexins evolved in the animal kingdom (for reviews see refs 2-8).

Despite such progress, there is still a gap in understanding between the functional properties of synaptic exocytosis and the molecular features of the key proteins. Modern electrophysiological ${ }^{9,10}$ and imaging approaches ${ }^{11-13}$ provided a wealth of information about the number of docked and primed vesicles, the exchange rates of vesicles between different pools, their release probabilities, their kinetics of exocytosis, and the dependence of exocytosis on calcium. Thus, detailed job descriptions for the underlying molecular machines are available. However, whereas genetic perturbations were instrumental in defining the basic functions of the key proteins, it often proved difficult to assign them to a specific step in the exocytotic pathway. For instance, Munc18, synaptotagmin and even the SNAREs were shown to function in docking as well as in priming and triggering. Conversely, specific steps such as docking are controlled by multiple proteins (see refs 7 and 11 for a more detailed discussion). It also often proved difficult to reconcile the physiological effects of the perturbations with the physicochemical properties of the proteins. Thus, the molecular mechanisms responsible for the attachment of synaptic vesicles to the active zone, for the activation of the release machinery, and for calcium triggering of exocytosis on a millisecond timescale are only slowly emerging.

\section{SNARE proteins, the engine of membrane fusion}

The synaptic proteins synaptobrevin (also referred to as VAMP), syntaxin 1 and SNAP-25 belong to the SNARE protein family. Their

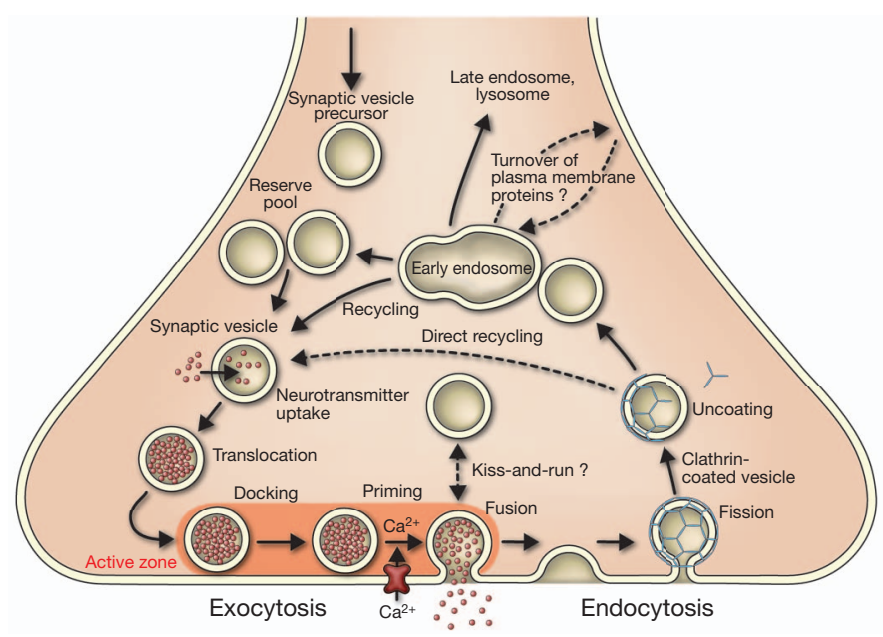

Figure $1 \mid$ Trafficking pathways in the nerve terminal. Synaptic vesicles are filled with neurotransmitter and stored in the cytoplasm. Active vesicles are translocated to release sites in the active zone where they dock. Priming involves all steps required to acquire release readiness of the exocytotic complex. Although usually assumed to occur after docking, priming and even triggering may precede docking during sustained activity, resulting in immediate fusion of an arriving vesicle. After exocytosis, the vesicle proteins probably remain clustered and are then retrieved by endocytosis. Despite some lingering controversies, consensus is emerging that retrieval is generally mediated by clathrin-mediated endocytosis. After clathrin uncoating, synaptic vesicles are regenerated within the nerve terminal, probably involving passage through an endosomal intermediate. Actively recycling vesicles are in slow exchange with the reserve pool. See text for more details. 
defining feature is an extended coiled-coil stretch, which is referred to as a SNARE motif and falls into four subtypes, referred to as Qa, Qb, Qc and R-SNARE motif (for example ref. 14). In syntaxin, synaptobrevin and in most other SNAREs the SNARE motifs are connected by a short linker to a carboxy-terminal transmembrane region (TMR). SNAP-25 deviates from this general structure: here two SNARE motifs (Qb and Qc) are connected by a linker that is palmitoylated, whereas a TMR is lacking. Whereas synaptobrevin and SNAP-25 do not carry any other domains, syntaxin possesses an amino-terminal domain consisting of an antiparallel three-helix bundle, termed the Habc domain ${ }^{15,16}$, connected to the SNARE motif by a flexible linker. Positioned N-terminally to the Habc domain is a short stretch that ends in the so-called N-peptide (see Fig. 2).

SNAREs undergo a regulated assembly-disassembly cycle that is energized by the AAA+-ATPase NSF. Synaptobrevin is a synaptic vesicle protein, whereas syntaxin 1 and SNAP-25 are localized in the presynaptic plasma membrane. On contact, the SNAREs associate in trans at the N-terminal ends of the SNARE motifs. A tight bundle of four parallel $\alpha$-helices is formed, each contributed by a different SNARE motif ${ }^{17,18}$, which progresses towards the C-terminal membrane anchors ('zippering'), thus pulling the membranes tightly together ${ }^{19}$. Assembly is associated with a huge release of energy that is used to initiate membrane fusion $^{20,21}$. After fusion, the ternary SNARE complex resides in the plasma membrane in the low-energy cis configuration and is disassembled by NSF in conjunction with its SNAP cofactor. Next, synaptobrevin is endocytosed and recycled, thus being able to participate in another round of exocytosis (for reviews see refs 2-8).

Despite the elegant simplicity and experimental support ${ }^{22}$ of the zippering model, SNARE assembly proved to be an unexpectedly complex reaction, and there is still a lot to learn. In vitro, isolated SNARE motifs are unfolded but assemble into diverse homo- and hetero-oligomers that all are at least partially helical (reviewed in refs 4 and 8). For instance, SNAP-25 can bind sequentially two syntaxin molecules, thus blocking the binding site of synaptobrevin ${ }^{23}$. Furthermore, syntaxin rapidly switches between an active open conformation and an inactive closed conformation in which the Habc domain folds against the N-terminal part of the SNARE motif ${ }^{24,25}$. Such conformational dynamics and kinetic trapping of off-pathway intermediates explains why in vitro assembly of the ternary complex, although highly exergonic, lasts hours, far too slow to mediate fast exocytosis. On the other hand, if a complex of SNAP-25 and syntaxin
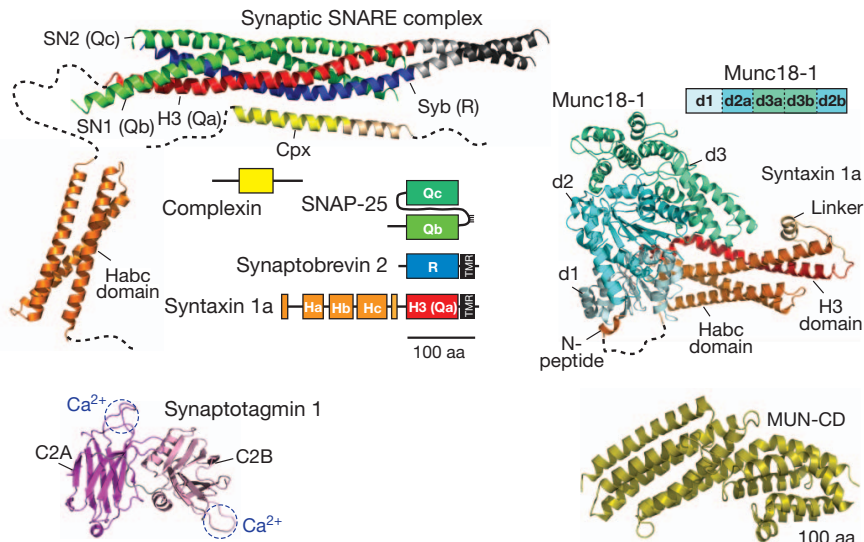

Synaptotagmin 1 差-



Figure 2 Schematic depictions of domain structures and crystal structures of core proteins of the neuronal fusion machine. The dashed lines between the N-peptide $(\mathrm{N})$ and Habc domain represent flexible regions in syntaxin. For synaptotagmin the two $\mathrm{Ca}^{2+}$ binding sites are indicated. Note that the domain structure of the large multi-domain protein Munc13 is shown five times smaller than those of the other domain structures. A high-resolution structure was obtained for the C-terminal half of the MUN domain. See text for details. The data for structures are from: Munc13-1 (C and D subdomains $)^{48}$, Synaptotagmin 1 (C2A and C2B domain) ${ }^{117}$, Munc18-1 (blue-green, in complex with syntaxin $($ red $))^{32}$, Habc domain ${ }^{16}$, SNARE complex ${ }^{18}$, complexin ${ }^{63}$. aa, amino acid. with a free $\mathrm{N}$-terminal binding site for synaptobrevin is stabilized, SNARE assembly is accelerated by orders of magnitude ${ }^{26}$. The central problem is to delineate precisely the assembly pathway and to understand how the SNARE molecules are channelled along this pathway by regulatory proteins to execute fusion efficiently. Four proteins, each representing a small protein family, have emerged as such key regulators: Munc18 and Munc13 that prepare the SNARE engine for assembly, and synaptotagmin and complexin that govern calcium-dependent triggering.

\section{Priming the SNARE engine}

UNC-18 and UNC-13, the Caenorhabditis elegans orthologues of Munc18 and Munc13, respectively, were originally identified by S. Brenner in his classical screen uncovering genes involved in movement $^{27}$. Deletion of either Munc18 (ref. 28) or Munc13 (ref. 29) and their respective orthologues ${ }^{30,31}$ completely inhibits neuronal exocytosis. Munc18 belongs to the conserved family of SM proteins. It possesses an arch-shaped architecture with a central cavity for high-affinity binding to syntaxin-1 (refs 32, 33). By contrast, the large Munc13s belong to the CATCHR protein family ${ }^{34}$. Munc13 also binds to syntaxin-1 but only with moderate affinity ${ }^{35,36}$. Both proteins are involved in setting up the SNAREs for assembly and perhaps in guiding them through the initial part of the assembly pathway, but it is still not understood how exactly they operate, how many copies are required to carry out the reaction, and how the extraordinary phenotypes of the knockouts can be mechanistically explained.

\section{Munc18}

For many years, the molecular mechanism of Munc18 has been shrouded by a paradox because it locks syntaxin-1 in a closed conformation $^{24,33}$ (Fig. 2), in which syntaxin cannot enter SNARE complexes. Such inhibition is difficult to reconcile with the complete loss of exocytosis in deletion mutants, which suggests exactly the opposite, namely that SNARE zippering is absolutely dependent on Munc18. Indeed, Munc18 seems to be an oddity because other SM proteins, despite high structural similarity, bind instead tightly to the N-peptide of their cognate syntaxins, involving a binding site on the surface of the SM protein. This binding mode would enable these syntaxins to remain open, with SNARE assembly not being inhibited, whereas syntaxin-1 would need to be opened in the case of Munc18. To reconcile these discrepancies, it was proposed that binding of SM proteins to syntaxins, whether via the $\mathrm{N}$-peptide or the Habc domain, merely serves to recruit the SM protein to the prospective fusion site. The SM proteins are then handed over to the SNARE motifs where they promote nucleation and/or zippering (for reviews see refs 3 and 37).

Recently, it has been recognized that SM proteins, including Munc18, generally bind to their respective syntaxins using both of the spatially distinct binding sites, but with different relative affinities ${ }^{32,38,39}$. In fact, the two binding sites seem to act together in controlling SNARE complex formation ${ }^{32}$. This sheds new light on the paradox, as full 'opening' of syntaxin may not be required for gating entry into SNARE complexes. In support of this view, a syntaxin mutant originally thought to be constitutively open (LE mutant) ${ }^{24}$ is now known to bind Munc18 via both sites in an at least partially closed conformation, but without inhibiting formation of SNARE complexes ${ }^{32,36}$. Indeed, when expressed as the only syntaxin 1 variant, the LE mutant results in enhanced spontaneous exocytosis, supporting that under resting conditions it is more reactive with respect to SNARE binding ${ }^{40}$.

Thus it seems that binding of Munc18-1 to both the closed conformation and to the N-peptide of syntaxin 1a is an integral part of the pathway during which Munc18 guides syntaxin towards productive SNARE complex formation. Perhaps Munc18 first keeps syntaxin closed and inactive, thus preventing premature SNARE assembly, but allows for synchronization of a subsequent (calcium-dependent?) activation step (see for example ref. 3 ).

Despite such progress, it is still unclear why Munc18 is essential for efficient SNARE nucleation. Reconstitution experiments involving 
liposomes suggest that Munc18 participates in selecting the correct R-SNARE helix and thus guides nucleation of the ternary complex (for example refs 41 and 42). It is unclear whether it then remains associated with the ternary complex ${ }^{43}$ as also suggested for other SM proteins (for example ref. 37 ) or dissociates upon zippering ${ }^{44}$. In the latter case Munc18 might be interacting with a trans-SNARE complex only during its initiation, whereas progression of zippering would cause syntaxin to fully open, thus driving off Munc18.

\section{Munc13}

Munc13s are modular proteins sharing a conserved C-terminal region containing a phorbol-ester-binding $\mathrm{C} 1$ domain and two calciumbinding C2 domains that flank a larger, so-called MUN domain (Fig. 2, reviewed in ref. 45). Expression of the MUN domain alone partially rescues the total arrest of exocytosis in neurons lacking Munc $13 \mathrm{~s}^{46}$, identifying it as a key functional element of the protein. MUN domains are shared with the proteins BAP3 and CAPS (Unc31) and with other proteins in most eukaryotes ${ }^{47}$. The MUN domain is structurally strikingly similar to other CATCHR family members ${ }^{48}$ that work in various trafficking steps. These proteins form elongated arrays of stacked $\alpha$-helical bundles with flexible hinge regions, which tether transport vesicles to the site of fusion. It is conceivable that the conserved MUN domain serves as binding platforms that arrange the core fusion machinery, whereas the $\mathrm{C} 1$ and $\mathrm{C} 2$ domains mediate fine-tuning of its membrane recruitment, a feature ideally suited to $\mathrm{Ca}^{2+}$-regulated secretion.

CATCHR complexes are also thought to enable SNARE assembly, although their interplay with SNARE proteins seems to vary. For instance, Munc13 may participate directly by unlocking syntaxin from the grip of Munc18, because in C. elegans, the LE mutant of syntaxin (that is not inhibited by Munc18 binding) partially rescues neurotransmitter release in the absence of UNC-13 (refs 49 and 50). Furthermore, recent experiments have shown that the isolated MUN domain accelerates the transition of syntaxin-1 from the Munc18-1 complex to the SNARE complex ${ }^{36}$. It should be kept in mind, however, that the LE mutant also partially rescues the block of exocytosis caused by deletion of RIM (also known as UNC-10) in C. elegans ${ }^{51}$. RIM serves as central organizer of the active zone. It forms a tripartite complex with the $\mathrm{N}$-terminal C2A domain of Munc13 and the small vesicular GTPases RAB3 and RAB27, thus orchestrating the attachment site of synaptic vesicles (reviewed in ref. 2).

\section{$\mathrm{Ca}^{2+}$-dependent triggering starts the SNARE engine}

In contrast to the basic fusion reaction that is carried out by conserved proteins traced back to an ancient eukaryotic machine, the unique features of calcium-triggered exocytosis are primarily encoded in specialized proteins. Of these, synaptotagmins I, II and IX constitute the dominant calcium sensors whose deletion results in a complete loss of fast, calcium-triggered exocytosis (reviewed in refs 52-54). However, asynchronous (that is, slower) calcium-dependent release persists, showing that other calcium-binding proteins are involved, with candidates including other synaptotagmin isoforms or related proteins such as Doc2 (refs 55-57). Furthermore, complexins I and II are involved in triggering: deletion of complexins strongly reduces calcium-evoked exocytosis, whereas both stimulatory and inhibitory effects were observed on spontaneous release (for example see refs 58 and 59).

\section{Synaptotagmins}

The neuronal synaptotagmins are anchored to synaptic vesicles by a single TMR. Characteristic features of the synaptotagmins are two C2 domains, called $\mathrm{C} 2 \mathrm{~A}$ and $\mathrm{C} 2 \mathrm{~B}$, that are connected to the membrane as well as to each other by flexible linkers. C2 domains are rigid, ovalshaped $\beta$-sandwiches that possess a cluster of calcium-binding loops, serving as partial coordination site for two (C2A) or three (C2B) calcium ions. In the presence of calcium, the $\mathrm{C} 2$ domains bind to membranes containing acidic phospholipids that complete the calcium coordination sites. In addition, the C2B domain contains a spatially separated basic patch that steers the domain to membranes enriched in phosphatidylinositol $(4,5)$ bisphosphate $\left(\mathrm{PI}(4,5) \mathrm{P}_{2}\right)$. Membrane binding is primarily electrostatic and rapidly reversed by chelating calcium or increasing the ionic strength. Furthermore, the synaptotagmin C2 domains bind to syntaxin alone or syntaxin-containing SNARE complexes (for example see refs 60 and 61). Although binding occurs in the absence of calcium, it appears to be influenced by calcium (reviewed in refs 54 and 62).

\section{Complexins}

Complexins are small cytoplasmic proteins that bind via a central helix to a groove on the surface of the SNARE complex, which is formed by the helices of syntaxin and synaptobrevin ${ }^{63,64}$ (Fig. 2). Because SNARE binding is required for their physiological action, complexins can only exert their function once SNAREs are at least partially assembled, placing them into the reaction sequence after zippering is initiated. Intriguingly, the central helix is not sufficient for complexin function. Rather, the N-terminal end pointing towards the membrane is needed for facilitation of fusion, whereas the regions flanking the central helix seem to have an inhibitory role. To accommodate the presumed dual stimulatory and inhibitory role of complexin, two alternative molecular mechanisms are discussed ${ }^{3,9,65-67}$. First, binding to the surface of the SNARE complex may promote initiation and progression of zippering, for example, by stabilizing partially zippered SNARE complexes and sensitizing them to activation by synaptotagmin ('super-priming') (for example see ref. 58). Second, complexin acts as a clamp that blocks progression of SNARE-zippering, presumably by competing directly with synaptobrevin binding in the C-terminal part of the SNARE complex (for example see refs 59 and 68). The clamp is released upon calcium triggering, probably by synaptotagmin (see below) because complexins do not bind calcium.

\section{Two models explain the action of calcium}

Despite many years of research, it is still controversial as to how calcium influx brings about the extraordinary and highly cooperative acceleration of exocytosis. To some extent this is owing to the fact that the molecular status of a docked and primed vesicle, ready to respond to calcium by exocytosis in less than a millisecond, is not known with certainty.

Most authors seem to agree that SNAREs are already partially zippered in this state, with full zippering being prevented either by an energy barrier in the fusion pathway that the SNAREs alone cannot overcome (for example, electrostatic repulsion, transition towards a stalk intermediate, see below), and/or by an interfering protein, with prime candidates being complexins and/or synaptotagmins (Fig. 3, pathway I). During this state, Munc18, and perhaps also Munc13, may still be bound to the complex. It is debated whether such a complex is strained, that is, storing energy that is released during fusion, or whether it is relaxed, with the linkers connecting the zippered part of the complex to the membrane being flexible.

Calcium binding to synaptotagmin would trigger fusion either by activating (disinhibiting) the SNAREs or by lowering the activation energy barrier in the fusion pathway through membrane interactions. Accordingly, synaptotagmin may act by (1) disengaging from the SNAREs, thus relieving the block (fusion clamp model) ${ }^{69}$, (2) binding to the SNAREs, thus displacing the inhibitory complexin and/or promoting zippering ${ }^{59}$, (3) binding to the membrane directly adjacent to the partially complexed SNAREs, thus destabilizing the bilayer at the fusion site $^{70-72}$, (4) increasing curvature stress by displacing lipids in the monolayer of the plasma membrane facing the vesicle ${ }^{73,74}$, and (5) cross-linking the vesicle and the plasma membrane, thus accelerating fusion by charge compensation owing to the positive electrostatic potential of the $\mathrm{C} 2$ domains $^{75}$.

A wealth of evidence is invoked in support of a partially zippered and arrested SNARE complex; for instance, differential effects of SNARE mutations on fusion kinetics that affect nucleation and zippering, 
Recruitment of Munc18



Activation of the SNARE acceptor complex

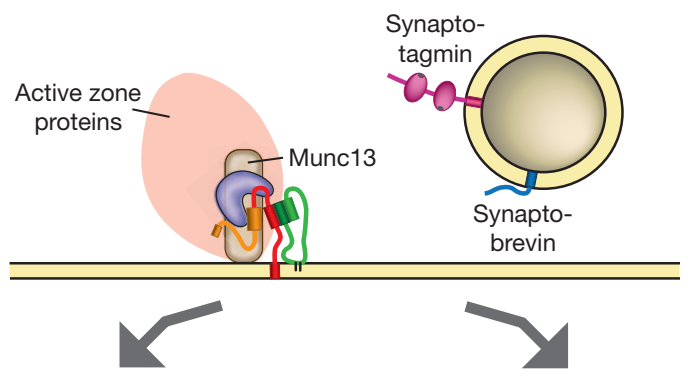

Priming I
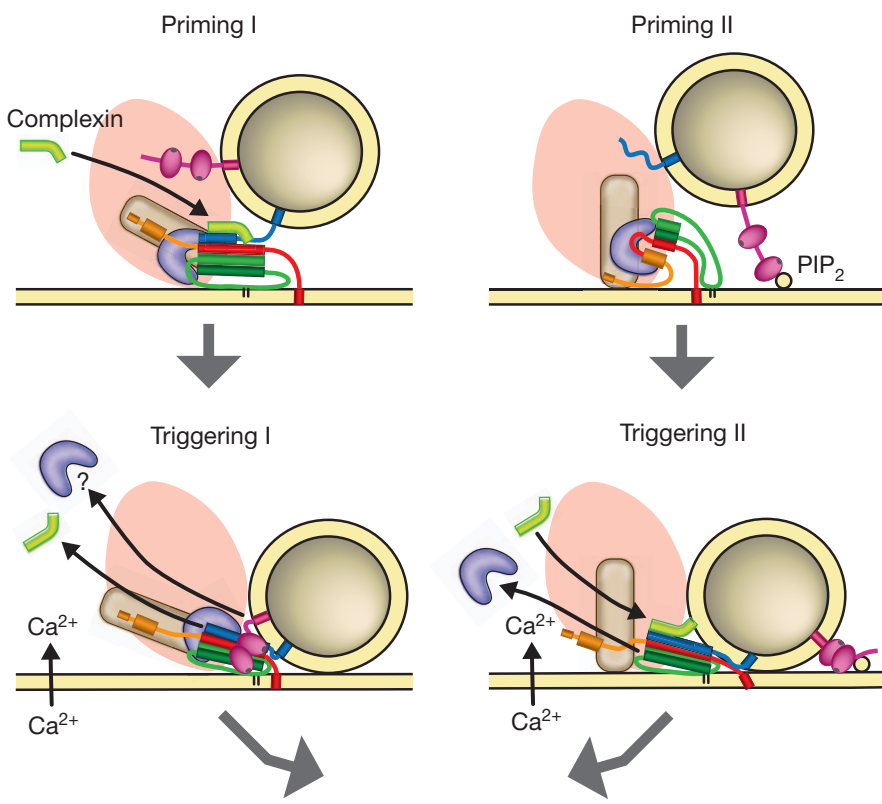

Triggering II

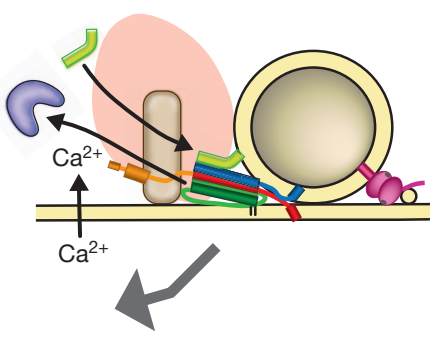

Fusion
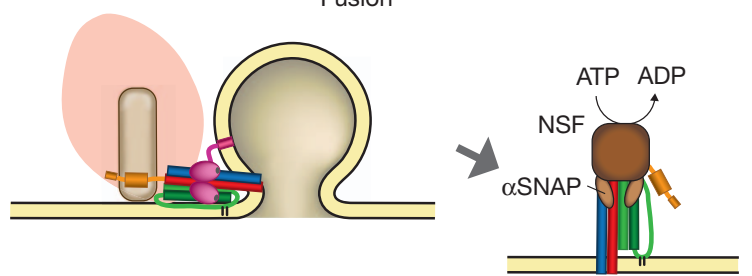

Figure 3 Alternative models describing the steps between priming and fusion. Priming I involves arrest of a partially zippered SNARE complex, here shown with bound Munc18, Munc13 and synaptotagmin. Calcium influx triggers binding of synaptotagmin to the SNARE complex and to the plasma membrane (involving $\mathrm{PI}(4,5) \mathrm{P}_{2}$, not shown here), associated with displacement of complexin and (possibly) Munc18 and/or Munc13. Priming II involves arrest after positioning of the vesicle with the aid of active zone components and (possibly) contact of synaptotagmin with $\mathrm{PI}(4,5) \mathrm{P}_{2}$ in the plasma membrane, but no contact between the SNAREs. $\mathrm{Ca}^{2+}$-triggering pulls the vesicle closer via synaptotagmin-mediated cross-linking, resulting in SNARE assembly, associated with full opening of syntaxin and displacement of Munc18, and binding of complexin. See text for details.

respectively (see for example refs 76-78). The model also allows for an integration of complexin into the fusion mechanism that needs at least partial SNARE assembly before it can bind and exert its action.
Furthermore, the model intuitively explains the fast fusion kinetics because only minor conformational rearrangements are required upon $\mathrm{Ca}^{2+}$-triggering, with all proteins already being correctly positioned for the final step.

On the other hand, there are problems with this model, which in our opinion have not been sufficiently appreciated. Experimentally, transcomplexes are difficult to capture. Similarly, despite hints, for example, from single-molecule experiments ${ }^{4,79}$, an effect of synaptotagmin and/or complexin on the rate of SNARE assembly has remained elusive. Most importantly, the mechanisms proposed for arresting SNARE zippering somewhere in the middle are difficult to reconcile with the fact that SNARE assembly proceeds along a steep downhill energy gradient. For instance, a C-terminal fragment of synaptobrevin forms in vitro a stable complex with SNAP-25 and syntaxin, thus blocking, like a brake shoe, the C-terminal portion from assembling as envisioned in the partially zippered model. However, full-length synaptobrevin is able to rapidly displace this fragment ${ }^{26}$ and, despite this additional energy barrier, to promote fusion in vitro even if only one of such partially inhibited SNARE complexes is involved ${ }^{80}$. None of the proposed factors (including complexin) binds with an affinity even remotely comparable to that of the synaptobrevin fragment, questioning their ability to influence the strongly exergonic zippering reaction. Furthermore, we consider it unlikely that the control of the neuronal SNAREs is exerted by tinkering with its structurally highly conserved engine core, that is, the helical bundle whose major features (structure, stability, foldingunfolding hysteresis) are remarkably similar between SNAREs in regulated and non-regulated trafficking steps ${ }^{8}$.

Similarly, a role of synaptotagmin in destabilizing membranes or inducing curvature stress is difficult to reconcile with $\mathrm{Ca}^{2+}$-dependent membrane binding being primarily electrostatic and reversible. Indeed, vesicle deformation in vitro requires saturation with $\mathrm{C} 2$ domaincontaining synaptotagmin fragments $\mathrm{s}^{73,74}$, whereas only few phospholipid molecules are expected to be displaced by binding of single $\mathrm{C} 2$ domains, hardly sufficient to create even local tension. Generally, agents increasing positive spontaneous curvature of the proximal monolayers inhibit rather than enhance fusion ${ }^{81}$, that is, exactly the opposite of what synaptotagmin is doing, requiring elaborate models of highly organized 'bulges' to explain promotion of fusion intermediates ${ }^{82}$.

Recently, an alternative scenario for the docked and primed state has been envisaged that, despite being far from proven, we consider as an interesting alternative, as it overcomes several of the problems outlined above $^{70,83}$ (Fig. 3, pathway II, see also ref. 40). Supported by recent electron tomography data showing that docked vesicles appear to be a few nanometres away from the plasma membrane ${ }^{11,12}$, it assumes that SNAREs do not connect in trans before the arrival of the calcium signal. Rather, the interaction of the vesicle with the active zone components (most notably Munc13 and RIM) would precisely position the vesicle on top of a patch of plasma membrane containing activated SNARE acceptor complexes, probably complexed with Munc18. In this state, vesicle-bound synaptotagmin may be already in contact with the plasma membrane, either by (calcium-independent) binding to the SNAREs or by loosely binding to $\mathrm{PI}(4,5) \mathrm{P}_{2}$ patches colocalizing with syntaxin clusters. Calcium influx would trigger membrane binding and cross-linking of the vesicle and plasma membrane, thus nudging them a bit clo$\operatorname{ser}^{75,83,84}$, sufficient to allow for rapid binding of synaptobrevin to the acceptor complex ${ }^{78}$. Once nucleation is triggered, the SNAREs quickly progress through zippering and fusion.

This model places the entire control of the neuronal fusion machine upstream of SNARE nucleation, which has important consequences for our understanding of the partial reactions. Most importantly, it changes the view of SNARE function. Accordingly, SNAREs act as 'single shot' devices that, once nucleation is triggered, are unstoppable and flash through assembly to bring about fusion. 'Misfiring' of SNAREs (assembly without fusion) probably only occurs rarely, if at all, but is likely to increase in mutants affecting zippering ${ }^{85}$. Also, it is possible that nucleation triggers the displacement of Munc18 and other factors (such 
as Munc13), thus allowing the SNAREs to carry out the work unhindered by bulky bound proteins. Such a simple and highly efficient mode of operation may explain why the SNARE engine was so successful in evolution. The proposed function for synaptotagmin is in line with the function of $\mathrm{C} 2$ domains in other proteins such as protein kinase $\mathrm{C}$-they operate as electrostatic switches ${ }^{62}$ mediating calcium-dependent rapid and reversible membrane binding.

The model also elegantly explains why solutions of high osmolarity (usually sucrose) trigger calcium-independent exocytosis of the readily releasable pool ${ }^{86}$ : the resulting water efflux creates negative pressure that draws docked vesicles closer to the plasma membrane, triggering SNARE firing. Furthermore, any destabilization of the overall architecture of the docking site, which increases Brownian fluctuations of the vesicle, would cause occasional spontaneous firing of SNAREs, which may explain changes in spontaneous release rates upon deletion or overexpression of some proteins (for a detailed discussion see for example refs 9 and 57).

Finally, the model provides for a fresh look at the molecular basis of the high cooperativity of calcium-triggered fusion. At non-saturating calcium concentrations, synaptotagmin binding may be less tight or transient, perhaps undergoing rapid and repetitive 'on-off' cycles, resulting in vesicle jittering. Accordingly, the probability for SNARE nucleation/firing would be reduced. Such a scenario may also explain the function of complexin, which is otherwise more difficult to integrate into this model. Complexin may increase the frequency of successful nucleation events by stabilizing correctly oriented syntaxin-synaptobrevin alignments. This hypothesis is in line with the ability of complexin to bind to SNARE complexes with fast, diffusion-limited kinetics ${ }^{87}$.

\section{Fusion-interplay between proteins and lipids}

In the final step of exocytosis the vesicle membrane fuses with the plasma membrane. The merger of two bilayers involves non-bilayer intermediates at the contact site that ultimately develop into the opening of an aqueous channel, termed a fusion pore. During fusion the hydrophobic barrier separating the cytoplasm from both the vesicle content and the extracellular space must remain intact.

Key issues concerning the molecular rearrangements of proteins and membrane lipids along the fusion pathway are unresolved. Popular models requiring an oligomeric ring of SNARE complexes surrounding the prospective fusion pore as intermediate cannot be maintained in view of the fact that only one to two (or three) SNARE complexes are sufficient for fusion both in vitro and in vivo ${ }^{80,88-90}$. Intriguingly, in vitro fusion can be mediated by trans assembly of artificially engineered molecules mimicking SNARE-zippering (even DNA) as long as they possess membrane anchors (for example see refs 91-94). Such a lack of structural specificity in catalysis is indeed a hallmark of membrane fusion, and it is likely that considerable structural variety is tolerated along the fusion pathway. This helps to explain why unrelated classes of fusion proteins evolved in parallel to the SNAREs, such as those fusing cells $^{95}$, viruses ${ }^{96}$, mitochondria ${ }^{97}$ or the endoplasmic reticulum ${ }^{98}$.

The stalk hypothesis, first developed 30 years ago ${ }^{99}$, describes membrane fusion as an ordered sequence of steps initiated by an hourglassshaped intermediate (the fusion stalk), followed by a hemifusion diaphragm and subsequent rupture, resulting in the formation of a fusion pore (Fig. 4). Indeed, stalk-like intermediates can be induced as a separate phase under mild conditions ${ }^{100-102}$. However, the energy landscape as well as the intermediate molecular structures along the fusion pathway is unclear.

Originally, the energy profile was modelled on the basis of the elastic properties of membranes, with the curvature stress of the intermediate model structures defining transition-state energies. However, these energies were unrealistically high, and molecular parameters were invoked to lower the energies (for review see ref. 103). More recently, coarse-grain or even atomistic simulations of fusion have provided detailed scenarios for intermediate structures (Fig. 4), with consequences for the energy landscape. For instance, it has been suggested that 'splaying' of phospholipid tails may form the first hydrophobic
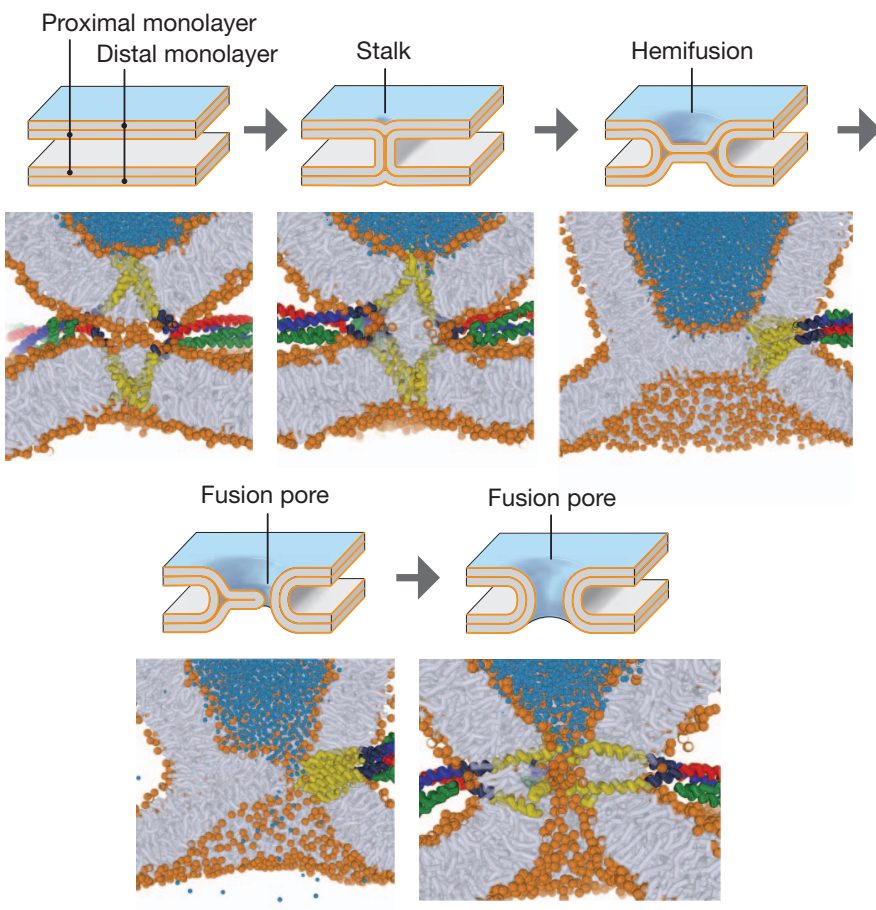

Figure $4 \mid$ Transition states during membrane fusion. Intermediates of the fusion pathway. The top drawings represent intermediate states of the membrane along the pathway as predicted by the elastic theory. Below, snapshots of intermediate states of a simulation of SNARE-mediated fusion are shown, which, although roughly corresponding to the elastic model, differ in detail and in their energy predictions (adapted from ref. 109, courtesy of J. Rissellada and H. Grubmüller).

connection between the membranes ${ }^{104}$ from where stalk formation proceeds downhill an energy gradient. Furthermore, the enhanced fusogenicity of curved membranes can also be explained by the hydrophobic effect: owing to the increased spacing of the hydrophilic head groups the membrane surface is more hydrophobic. Lipid splaying requires the membranes to be at a critical distance of less than $1 \mathrm{~nm}$ (Fig. 4, see ref. 105 for a more detailed discussion).

These considerations have important consequences for the mechanism of SNARE-catalysed fusion. Certainly, zippering of the four-helix bundle brings the membranes in close proximity, but the question is how the SNAREs promote stalk formation and subsequent intermediate structures. If the main energy barrier is contributed by curvature stress $^{106}$, stiffness of the linkers connecting SNARE motifs and TMRs is essential for transmitting stress to the membranes. Indeed, mutagenesis of the linkers generally reduces fusion efficacy (see for example ref. 107), and at least syntaxin seems to have a stiff linker as a monomer ${ }^{108}$. On the other hand, if close proximity, water removal, increase of local hydrophobicity and lipid splaying form the main energy barrier, bending stiffness of the SNARE linkers may not be as relevant, a view suggested by recent simulation studies ${ }^{109}$. Instead, the pulling force exerted during zippering may drag the TMRs along with some phospholipids slightly out of the membrane, thus initiating phospholipid splaying once the critical distance has been reached.

What are the next steps? Transient hemifusion intermediates (experimentally defined, for example, by lipid mixing in the absence of content mixing) are observed upon SNARE-mediated fusion of liposomes, suggesting hemifusion as a metastable intermediate (for example see refs 110-114). However, it is experimentally difficult to differentiate between stalk and hemifusion intermediates. Hemifusion constitutes the lateral expansion of a stalk, leading to the formation of a hemifusion diaphragm (Fig. 4). It remains to be seen whether such diaphragms represent intermediates along the fusion pathway or whether they are dead-ends as previously suggested for viral fusion proteins (see ref. 115 
for a review). In any case, the job of the SNAREs is not finished before the initial opening of the fusion pore, with interactions between the linkers as well as the TMRs probably being involved ${ }^{116}$.

\section{Conclusion}

The molecular basis of synaptic exocytosis has fascinated scientists for decades. Since the initial discovery of quantal release in the 1950s by Katz and colleagues, and the elucidation of the synaptic vesicle-recycling pathway by Heuser and Ceccarelli in the 1970s, we have come a long way in deciphering the steps of the vesicle cycle at an increasingly detailed level. Although we have focused here on only a few key components, the vesicle cycle is governed by hundreds of proteins, and there are still new proteins being put on the map. We are only beginning to understand the rules by which individual protein-protein interactions work together in supramolecular machines to yield the synaptic vesicle cycle that reliably operates millions of times. These machines assemble on demand and disassemble when the task is completed. They are highly robust, tolerate varying stoichiometries, flexible compositions and other disturbances, and are controlled by an array of regulators such as protein kinases and phosphatases. Advances in technologies such as super-resolution microscopy, single-molecule measurements, fluorescent reporters and cryo-electron tomography are all contributing to closing the gap between our understanding of partial reactions in vitro and the fascinating efficiency of the vesicle cycle in intact synapses.

1. Südhof, T. C. The synaptic vesicle cycle. Annu. Rev. Neurosci. 27, 509-547 (2004).

2. Südhof, T. C. \& Rizo, J. Synaptic vesicle exocytosis. Cold Spring Harb. Perspect. Biol. 3, a005637 (2011)

3. Südhof, T. C. \& Rothman, J. E. Membrane fusion: grappling with SNARE and SM proteins. Science 323, 474-477 (2009).

4. Brunger, A. T., Weninger, K., Bowen, M. \& Chu, S. Single-molecule studies of the neuronal SNARE fusion machinery. Annu. Rev. Biochem. 78, 903-928 (2009).

5. Wickner, W. \& Schekman, R. Membrane fusion. Nature Struct. Mol. Biol. 15, 658-664 (2008).

6. Rizo, J. \& Rosenmund, C. Synaptic vesicle fusion. Nature Struct. Mol. Biol. 15, 665-674 (2008).

7. Verhage, M. \& Sørensen, J. B. Vesicle docking in regulated exocytosis. Traffic 9 , 1414-1424 (2008)

8. Jahn, R. \& Scheller, R. H. SNAREs-engines for membrane fusion. Nature Rev. Mol. Cell Biol. 7, 631-643 (2006).

9. Sørensen, J. B. Conflicting views on the membrane fusion machinery and the fusion pore. Annu. Rev. Cell Dev. Biol. 25, 513-537 (2009).

10. Neher, E. \& Sakaba, T. Multiple roles of calcium ions in the regulation of neurotransmitter release. Neuron 59, 861-872 (2008).

11. Siksou, L., Triller, A. \& Marty, S. Ultrastructural organization of presynaptic terminals. Curr. Opin. Neurobiol. 21, 261-268 (2011).

12. Fernáandez-Busnadiego, R. et al. Insights into the molecular organization of the neuron by cryo-electron tomography. J. Electron Microsc. (Tokyo) 60 (suppl. 1), S137-S148 (2011)

13. Sigrist, S. J. \& Sabatini, B. L. Optical super-resolution microscopy in neurobiology. Curr. Opin. Neurobiol. 22, 86-93 (2012)

14. Kloepper, T. H., Kienle, C. N. \& Fasshauer, D. An elaborate classification of SNARE proteins sheds light on the conservation of the eukaryotic endomembrane system. Mol. Biol. Cell 18, 3463-3471 (2007).

15. Fernandez, I. et al. Three-dimensional structure of an evolutionarily conserved $\mathrm{N}$-terminal domain of syntaxin 1A. Cell 94, 841-849 (1998).

16. Lerman, J. C., Robblee, J., Fairman, R. \& Hughson, F. M. Structural analysis of the neuronal SNARE protein syntaxin-1A. Biochemistry 39, 8470-8479 (2000).

17. Sutton, R. B., Fasshauer, D., Jahn, R. \& Brunger, A. T. Crystal structure of a SNARE complex involved in synaptic exocytosis at 2.4 A resolution. Nature 395 , 347-353 (1998).

18. Stein, A., Weber, G., Wahl, M. C. \& Jahn, R. Helical extension of the neuronal SNARE complex into the membrane. Nature 460, 525-528 (2009). This paper describes the X-ray structure of the synaptic SNARE complex with transmembrane regions.

19. Hanson, P. I., Heuser, J. E. \& Jahn, R. Neurotransmitter release - four years of SNARE complexes. Curr. Opin. Neurobiol. 7, 310-315 (1997).

20. Li, F. et al. Energetics and dynamics of SNAREpin folding across lipid bilayers. Nature Struct. Mol. Biol. 14, 890-896 (2007).

21. Wiederhold, K. \& Fasshauer, D. Is assembly of the SNARE complex enough to fuel membrane fusion? J. Biol. Chem. 284, 13143-13152 (2009).

22. Weber, T. et al. SNAREpins: minimal machinery for membrane fusion. Cell 92, 759-772 (1998).

23. Fasshauer, D. \& Margittai, M. A Transient N-terminal interaction of SNAP-25 and syntaxin nucleates SNARE assembly. J. Biol. Chem. 279, 7613-7621 (2004).

24. Dulubova, I. et al. A conformational switch in syntaxin during exocytosis: role of munc18. EMBO J. 18, 4372-4382 (1999).
25. Margittai, M. et al. Single-molecule fluorescence resonance energy transfer reveals a dynamic equilibrium between closed and open conformations of syntaxin 1. Proc. Natl Acad. Sci. USA 100, 15516-15521 (2003).

26. Pobbati, A. V., Stein, A. \& Fasshauer, D. N- to C-terminal SNARE complex assembly promotes rapid membrane fusion. Science 313, 673-676 (2006).

27. Brenner, S. The genetics of Caenorhabditis elegans. Genetics 77, 71-94 (1974).

28. Verhage, M. et al. Synaptic assembly of the brain in the absence of neurotransmitter secretion. Science 287, 864-869 (2000).

29. Varoqueaux, F. et al. Total arrest of spontaneous and evoked synaptic transmission but normal synaptogenesis in the absence of Munc13-mediated vesicle priming. Proc. Natl Acad. Sci. USA 99, 9037-9042 (2002).

30. Richmond, J. E., Davis, W. S. \& Jorgensen, E. M. UNC-13 is required for synaptic vesicle fusion in C. elegans. Nature Neurosci. 2, 959-964 (1999).

31. Aravamudan, B., Fergestad, T., Davis, W. S., Rodesch, C. K. \& Broadie, K. Drosophila UNC-13 is essential for synaptic transmission. Nature Neurosci. 2, 965-971 (1999).

32. Burkhardt, P., Hattendorf, D. A., Weis, W. I. \& Fasshauer, D. Munc18a controls SNARE assembly through its interaction with the syntaxin $\mathrm{N}$-peptide. EMBO J. 27, 923-933 (2008).

This paper reports that Munc18-1 interacts with two spatially separated binding sites of syntaxin-1a.

33. Misura, K. M. Scheller, R. H. \& Weis, W. I. Three-dimensional structure of the neuronal-Sec1-syntaxin 1a complex. Nature 404, 355-362 (2000).

34. Yu, I. M. \& Hughson, F. M. Tethering factors as organizers of intracellular vesicular traffic. Annu. Rev. Cell Dev. Biol. 26, 137-156 (2010).

35. Betz, A., Okamoto, M., Benseler, F. \& Brose, N. Direct interaction of the rat unc-13 homologue Munc13-1 with the N terminus of syntaxin. J. Biol. Chem. 272, 2520-2526 (1997).

36. Ma, C., Li, W., Xu, Y. \& Rizo, J. Munc13 mediates the transition from the closed syntaxin-Munc18 complex to the SNARE complex. Nature Struct. Mol. Biol. 18, 542-549 (2011).

37. Toonen, R. F. \& Verhage, M. Munc18-1 in secretion: Ionely Munc joins SNARE team and takes control. Trends Neurosci. 30, 564-572 (2007).

38. Furgason, M. L. et al. The N-terminal peptide of the syntaxin Tlg2p modulates binding of its closed conformation to Vps45p. Proc. Natl Acad. Sci. USA 106, 14303-14308 (2009).

39. Khvotchev, M. et al. Dual modes of Munc18-1/SNARE interactions are coupled by functionally critical binding to syntaxin-1 N terminus. J. Neurosci. $\mathbf{2 7}$ 12147-12155 (2007)

40. Gerber, S. H. et al. Conformational switch of syntaxin-1 controls synaptic vesicle fusion. Science 321, 1507-1510 (2008).

This paper and ref. 49 describe the complex phenotype of the LE mutant of syntaxin on docking and fusion of synaptic vesicles.

41. Rathore, S. S. etal. Syntaxin N-terminal peptide motif is an initiation factor for the assembly of the SNARE-Sec1/Munc18 membrane fusion complex. Proc. Natl Acad. Sci. USA 107, 22399-22406 (2010).

42. Shen, J., Tareste, D. C.. Paumet, F., Rothman, J.E. \& Melia, T. J. Selective activation of cognate SNAREpins by Sec1/Munc18 proteins. Cell 128, 183-195 (2007).

43. Xu, Y., Su, L. \& Rizo, J. Binding of Munc18-1 to synaptobrevin and to the SNARE four-helix bundle. Biochemistry 49, 1568-1576 (2010).

44. Zilly, F. E., Sørensen, J. B., Jahn, R. \& Lang, T. Munc18-bound syntaxin readily forms SNARE complexes with synaptobrevin in native plasma membranes. PLOS Biol. 4, e330 (2006)

45. Wojcik, S. M. \& Brose, N. Regulation of membrane fusion in synaptic excitationsecretion coupling: speed and accuracy matter. Neuron 55, 11-24 (2007).

46. Basu, J. et al. A minimal domain responsible for Munc13 activity. Nature Struct Mol. Biol. 12, 1017-1018 (2005).

47. Koch, H., Hofmann, K. \& Brose, N. Definition of Munc13-homology-domains and characterization of a novel ubiquitously expressed Munc13 isoform. Biochem. J. $349,247-253(2000)$

48. Li, W. et al. The crystal structure of a Munc13 C-terminal module exhibits a remarkable similarity to vesicle tethering factors. Structure 19, 1443-1455 (2011).

This crystal structure demonstrates that Munc13 is a member of the conserved CATCHR protein family involved in vesicle tethering.

49. Hammarlund, M., Palfreyman, M. T., Watanabe, S., Olsen, S. \& Jorgensen, E. M. Open syntaxin docks synaptic vesicles. PLoS Biol. 5, e198 (2007).

50. Richmond, J. E., Weimer, R. M. \& Jorgensen, E. M. An open form of syntaxin bypasses the requirement for UNC-13 in vesicle priming. Nature 412, 338-341 (2001).

51. Koushika, S. P. et al. A post-docking role for active zone protein Rim. Nature Neurosci. 4, 997-1005 (2001)

52. Kochubey, O., Lou, X. \& Schneggenburger, R. Regulation of transmitter release by $\mathrm{Ca}^{2+}$ and synaptotagmin: insights from a large CNS synapse. Trends Neurosci. 34, 237-246 (2011).

53. Pang, Z. P. \& Südhof, T. C. Cell biology of $\mathrm{Ca}^{2+}$-triggered exocytosis. Curr. Opin. Cell Biol. 22, 496-505 (2010)

54. Chapman, E. R. How does synaptotagmin trigger neurotransmitter release? Annu. Rev. Biochem. 77, 615-641 (2008).

55. Groffen, A. J. et al. Doc2b is a high-affinity $\mathrm{Ca}^{2+}$ sensor for spontaneous neurotransmitter release. Science 327, 1614-1618 (2010).

56. Yao, J., Gaffaney, J. D., Kwon, S. E. \& Chapman, E. R. Doc2 is a $\mathrm{Ca}^{2+}$ sensor required for asynchronous neurotransmitter release. Cell 147, 666-677 (2011)

57. Walter, A. M., Groffen, A. J., Sørensen, J. B. \& Verhage, M. Multiple $\mathrm{Ca}^{2+}$ sensors in secretion: teammates, competitors or autocrats? Trends Neurosci. 34, 487-497 (2011) 
58. Xue, M. et al. Binding of the complexin N terminus to the SNARE complex potentiates synaptic-vesicle fusogenicity. Nature Struct. Mol. Biol. 17, 568-575 (2010).

59. Yang, X., Kaeser-Woo, Y. J., Pang, Z. P., Xu, W. \& Südhof, T. C. Complexin clamps asynchronous release by blocking a secondary $\mathrm{Ca}^{2+}$ sensor via its accessory alpha helix. Neuron 68, 907-920 (2010).

60. Lai, A. L., Huang, H., Herrick, D. Z., Epp, N. \& Cafiso, D. S. Synaptotagmin 1 and SNAREs form a complex that is structurally heterogeneous. J. Mol. Biol. 405, 696-706 (2011)

61. Vrljic, M. etal. Molecular mechanism of the synaptotagmin-SNARE interaction in $\mathrm{Ca}^{2+}$-triggered vesicle fusion. Nature Struct. Mol. Biol. 17, 325-331 (2010).

62. Rizo, J., Chen, X. \& Arac, D. Unraveling the mechanisms of synaptotagmin and SNARE function in neurotransmitter release. Trends Cell Biol. 16, 339-350 (2006).

63. Chen, X. et al. Three-dimensional structure of the complexin/SNARE complex. Neuron 33, 397-409 (2002)

64. Bracher, A., Kadlec, J., Betz, H. \& Weissenhorn, W. X-ray structure of a neuronal complexin-SNARE complex from squid. J. Biol. Chem. 277, 26517-26523 (2002).

65. Brose, N. For better or for worse: complexins regulate SNARE function and vesicle fusion. Traffic 9, 1403-1413 (2008)

66. Stein, A. \& Jahn, R. Complexins living up to their name-new light on their role in exocytosis. Neuron 64, 295-297 (2009).

67. Neher, E. Complexin: does it deserve its name? Neuron 68, 803-806 (2010).

68. Kummel, D. et al. Complexin cross-links prefusion SNAREs into a zigzag array. Nature Struct. Mol. Biol. 18, 927-933 (2011)

69. Chicka, M. C., Hui, E., Liu, H. \& Chapman, E. R. Synaptotagmin arrests the SNARE complex before triggering fast, efficient membrane fusion in response to $\mathrm{Ca}^{2+}$. Nature Struct. Mol. Biol. 15, 827-835 (2008).

70. Stein, A., Radhakrishnan, A., Riedel, D., Fasshauer, D. \& Jahn, R. Synaptotagmin activates membrane fusion through a $\mathrm{Ca}^{2+}$-dependent trans interaction with phospholipids. Nature Struct. Mol. Biol. 14, 904-911 (2007).

71. Xue, M., Ma, C., Craig, T. K., Rosenmund, C. \& Rizo, J. The Janus-faced nature of the $\mathrm{C}_{2} \mathrm{~B}$ domain is fundamental for synaptotagmin-1 function. Nature Struct. Mol. Biol. 15, 1160-1168 (2008)

72. Lee, H. K. et al. Dynamic $\mathrm{Ca}^{2+}$-dependent stimulation of vesicle fusion by membrane-anchored synaptotagmin 1 . Science 328, 760-763 (2010).

73. Martens, S., Kozlov, M. M. \& McMahon, H. T. How synaptotagmin promotes membrane fusion. Science 316, 1205-1208 (2007).

74. Hui, E., Johnson, C. P., Yao, J., Dunning, F. M. \& Chapman, E. R. Synaptotagminmediated bending of the target membrane is a critical step in $\mathrm{Ca}^{2+}$-regulated fusion. Cell 138, 709-721 (2009).

75. Araç, D. et al. Close membrane-membrane proximity induced by $\mathrm{Ca}^{2+}$ dependent multivalent binding of synaptotagmin-1 to phospholipids. Nature Struct. Mol. Biol. 13, 209-217 (2006).

This study shows that synaptotagmin binds simultaneously to two membranes, bringing them into close proximity.

76. Sørensen, J.B. etal. Sequential N- to C-terminal SNARE complex assembly drives priming and fusion of secretory vesicles. EMBO J. 25, 955-966 (2006).

77. Walter, A. M., Wiederhold, K., Bruns, D., Fasshauer, D. \& Sørensen, J. B. Synaptobrevin N-terminally bound to syntaxin-SNAP-25 defines the primed vesicle state in regulated exocytosis. J. Cell Biol. 188, 401-413 (2010).

78. Wiederhold, K. et al. A coiled coil trigger site is essential for rapid binding of synaptobrevin to the SNARE acceptor complex. J. Biol. Chem. 285, 21549-21559 (2010)

79. Diao, J., Ishitsuka, Y. \& Bae, W. R. Single-molecule FRET study of SNAREmediated membrane fusion. Biosci. Rep. 31, 457-463 (2011).

80. van den Bogaart, G. et al. One SNARE complex is sufficient for membrane fusion. Nature Struct. Mol. Biol. 17, 358-364 (2010)

81. Chernomordik, L. V. \& Zimmerberg, J. Bending membranes to the task structural intermediates in bilayer fusion. Curr. Opin. Struct. Biol. 5, 541-547 (1995).

82. McMahon, H. T., Kozlov, M. M. \& Martens, S. Membrane curvature in synaptic vesicle fusion and beyond. Cell 140, 601-605 (2010).

83. van den Bogaart, G. et al. Synaptotagmin-1 may be a distance regulator acting upstream of SNARE nucleation. Nature Struct. Mol. Biol. 18, 805-812 (2011).

84. Kuo, W., Herrick, D. Z. \& Cafiso, D. S. Phosphatidylinositol 4,5-bisphosphate alters synaptotagmin 1 membrane docking and drives opposing bilayers closer together. Biochemistry 50, 2633-2641 (2011).

85. Schwartz, M. L. \& Merz, A. J. Capture and release of partially zipped trans-SNARE complexes on intact organelles. J. Cell Biol. 185, 535-549 (2009).

86. Rosenmund, C.\& Stevens, C. F. Definition of the readily releasable pool of vesicles at hippocampal synapses. Neuron 16, 1197-1207 (1996).

87. Pabst, S. et al. Rapid and selective binding to the synaptic SNARE complex suggests a modulatory role of complexins in neuroexocytosis. J. Biol. Chem. 277 7838-7848 (2002).

88. Mohrmann, R., de Wit, H., Verhage, M., Neher, E. \& Sørensen, J. B. Fast vesicle fusion in living cells requires at least three SNARE complexes. Science 330, 502-505 (2010).

Using a titration approach this study and ref. 89 reveal that neurotransmitter release requires only few SNARE complexes.

89. Sinha, R., Ahmed, S., Jahn, R. \& Klingauf, J. Two synaptobrevin molecules are sufficient for vesicle fusion in central nervous system synapses. Proc. Natl Acad. Sci. USA 108, 14318-14323 (2011)

90. Shi, L. et al. SNARE proteins: one to fuse and three to keep the nascent fusion pore open. Science 335, 1355-1359 (2012)
91. Chan, Y. H., van Lengerich, B. \& Boxer, S. G. Effects of linker sequences on vesicle fusion mediated by lipid-anchored DNA oligonucleotides. Proc. Natl Acad. Sci. USA 106, 979-984 (2009).

92. Simonsson, L., Jonsson, P., Stengel, G. \& Hook, F. Site-specific DNA-controlled fusion of single lipid vesicles to supported lipid bilayers. ChemPhysChem 11, 1011-1017 (2010)

93. Lygina, A. S., Meyenberg, K., Jahn, R. \& Diederichsen, U. Transmembrane domain peptide/peptide nucleic acid hybrid as a model of a SNARE protein in vesicle fusion. Angew. Chem. Int. Edn Engl. 50, 8597-8601 (2011).

94. Robson Marsden, H., Elbers, N. A., Bomans, P. H., Sommerdijk, N. A. \& Kros, A. A reduced SNARE model for membrane fusion. Angew. Chem. Int. Edn Engl. 48 2330-2333 (2009)

95. Avinoam, O. \& Podbilewicz, B. Eukaryotic cell-cell fusion families. Curr. Top. Membr. 68, 209-234 (2011)

96. Harrison, S. C. Viral membrane fusion. Nature Struct. Mol. Biol. 15, 690-698 (2008).

97. Westermann, B. Mitochondrial fusion and fission in cell life and death. Nature Rev. Mol. Cell Biol. 11, 872-884 (2010).

98. Moss, T. J., Daga, A. \& McNew, J. A. Fusing a lasting relationship between ER tubules. Trends Cell Biol. 21, 416-423 (2011)

99. Kozlov, M. M. \& Markin, V. S. Possible mechanism of membrane fusion [in Russian]. Biofizika 28, 242-247 (1983).

100. Yang, L. \& Huang, H. W. Observation of a membrane fusion intermediate structure. Science 297, 1877-1879 (2002)

101. Aeffner, S., Reusch, T., Weinhausen, B. \& Salditt, T. Structure, hydration barrier and curvature of membrane hemifusion stalks with varying lipid composition obtained by x-ray diffraction. Proc. Natl Acad. Sci. USA. (in the press).

102. Qian, S. \& Huang, H. W. A novel phase of compressed bilayers that models the prestalk transition state of membrane fusion. Biophys. J. 102, 48-55 (2012).

103. Chernomordik, L. V.\& Kozlov, M. M. Protein-lipid interplay in fusion and fission of biological membranes. Annu. Rev. Biochem. 72, 175-207 (2003).

104. Kinnunen, P. K. Fusion of lipid bilayers: a model involving mechanistic connection to HII phase forming lipids. Chem. Phys. Lipids 63, 251-258 (1992)

105. Risselada, H. J. \& Grubmuller, H. How SNARE molecules mediate membrane fusion: recent insights from molecular simulations. Curr. Opin. Struct. Biol. 22, 187-196 (2012)

106. Kozlov, M. M., McMahon, H. T. \& Chernomordik, L. V. Protein-driven membrane stresses in fusion and fission. Trends Biochem. Sci. 35, 699-706 (2010).

107. Kesavan, J., Borisovska, M. \& Bruns, D. v-SNARE actions during $\mathrm{Ca}^{2+}$-triggered exocytosis. Cell 131, 351-363 (2007).

\section{This study systematically measures the effect of extending the} juxtamembrane region of synaptobrevin on neurotransmitter release.

108. Knecht, V. \& Grubmuller, H. Mechanical coupling via the membrane fusion SNARE protein syntaxin 1A: a molecular dynamics study. Biophys. J. 84, 1527-1547 (2003).

109. Risselada, H. J., Kutzner, C. \& Grubmuller, H. Caught in the act: visualization of SNARE-mediated fusion events in molecular detail. ChemBioChem $\mathbf{1 2}$ 1049-1055 (2011)

Using coarse-grain simulations, the transition states involved in SNAREmediated membrane fusion are described on the basis of first principles.

110. Xu, Y., Zhang, F., Su, Z., McNew, J. A. \& Shin, Y. K. Hemifusion in SNARE-mediated membrane fusion. Nature Struct. Mol. Biol. 12, 417-422 (2005).

111. Wang, T., Smith, E. A., Chapman, E. R. \& Weisshaar, J. C. Lipid mixing and content release in single-vesicle, SNARE-driven fusion assay with $1-5 \mathrm{~ms}$ resolution. Biophys. J. 96, 4122-4131 (2009).

112. Giraudo, C. G. et al. SNAREs can promote complete fusion and hemifusion as alternative outcomes. J. Cell Biol. 170, 249-260 (2005)

113. Reese, C., Heise, F. \& Mayer, A. Trans-SNARE pairing can precede a hemifusion intermediate in intracellular membrane fusion. Nature 436, 410-414 (2005).

114. Chernomordik, L. V. \& Kozlov, M. M. Membrane hemifusion: crossing a chasm in two leaps. Cell 123, 375-382 (2005)

115. Chernomordik, L. V. \& Kozlov, M. M. Mechanics of membrane fusion. Nature Struct. Mol. Biol. 15, 675-683 (2008).

116. Laage, R., Rohde, J., Brosig, B. \& Langosch, D. A conserved membrane-spanning amino acid motif drives homomeric and supports heteromeric assembly of presynaptic SNARE proteins. J. Biol. Chem. 275, 17481-17487 (2000).

117. Fuson, K. L., Montes, M., Robert, J. J. \& Sutton, R. B. Structure of human synaptotagmin $1 \mathrm{C} 2 \mathrm{AB}$ in the absence of $\mathrm{Ca}^{2+}$ reveals a novel domain association. Biochemistry 46, 13041-13048 (2007).

Acknowledgements Work in the authors' laboratories was supported by grants from the National Institutes of Health (3P01GM072694-05S1) to D.F. and R.J., of the Swiss National Fond to D.F. (31003A_133055) and of the Deutsche

Forschungsgemeinschaft to D.F. (FA 297/3-1) and R.J. (SFB 803). The authors thank H. Grubmüller, E. Neher, J. Rissellada, G. van den Bogaart, M. Hernandez, J. Sørensen and J. Rizo for discussions and critical reading of the manuscript. We apologize to all colleagues whose work, although relevant, could not be mentioned and/or cited owing to space limitations.

Author Contributions Both authors wrote the manuscript.

Author Information Reprints and permissions information is available at www.nature.com/reprints. The authors declare no competing financial interests. Readers are welcome to comment on the online version of the paper. Correspondence should be addressed to R.J. (rjahn@gwdg.de). 